\title{
Formación inicial docente en el Modelo Educativo mexicano: perspectivas de directivos normalistas
}

\section{Pre-service teacher education in the Mexican Educational Model: Perspectives of teachers' college principals}

\section{DOI: $10.32870 /$ dse.v0i19.497}

Karen Patricia Rivera Ceseña*

Norma Leticia Carrillo Chávez**

Dolores Graciela Cordero Arroyo***

María del Ángel Vázquez Cruz****

\begin{abstract}
Resumen
El presente estudio, descriptivo y comparativo, tuvo como objetivo analizar las diferencias entre el apartado de formación inicial del Modelo Educativo 2016 (SEP, 2016a) y del mismo apartado del Modelo Educativo 2017 (SEP, 2017a) para contrastarlos con los planteamientos de los participantes del "Foro de Consulta de Directivos de Escuelas Normales" celebrado en agosto de 2016. El estudio se llevó a cabo con un enfoque cualitativo y se dividió en dos fases: 1) Análisis de contenido de los documentos oficiales y 2) Análisis de contenido de las mesas de trabajo del foro mencionado. Los resultados indican que si bien el Modelo 2017 recupera la mayoría de las ideas mencionadas por los participantes de los foros, hay otras que, aunque los directivos lo señalaron reiteradamente, no fueron referidas en el documento oficial de la SEP. Un ejemplo de esto es la implicación de las escuelas normales en la impartición de formación continua y la posibilidad de que los resultados del concurso de ingreso al servicio profesional docente puedan ser de utilidad para retroalimentar sus procesos formativos.
\end{abstract}

Palabras clave: educación normalista - reforma educativa - políticas públicas - modelos educativos directores

\section{Abstract}

The purpose of this descriptive and comparative study was to analyze the differences between the preservice teacher education section of the 2016 Educational Model (SEP, 2016a) and the same section of the 2017 Educational Model (SEP, 2017a), and to analyze its relationship with the participants' contributions

\footnotetext{
* Licenciada en Ciencias de la Educación. Asistente de investigación. Universidad Autónoma de Baja California. México.karenriverac17.10@ gmail.com

** Licenciada en Ciencias de la Educación. Asistente de investigación. Universidad Autónoma de Baja California. México. lety care@hotmail.com

*** Doctora en Filosofía y Ciencias de la Educación. Investigadora titular. Universidad Autónoma de Baja California. México. gcordero@uabc. edu.mx

**** Maestra en Ciencias Educativas. Docente. Universidad Autónoma de Baja California. México. vazquezm87@uabc.edu.mx
} 
to the "Consultation Forum of Principals of Normal Schools" held in August 2016. The study was conducted under a qualitative approach and was divided into two phases: 1) Content analysis of official documents and 2) Content analysis of the working groups of the "Forum of Consultation of Principals of Normal Schools". The results show that although the 2017 Model recovers most of the ideas mentioned by the participants of the forums, there are others that, although pointed out repeatedly by the principals, were not mentioned in the official document of the Ministry of Education. An example of this is the involvement of the teachers' colleges in the delivery of ongoing education and the possibility that the results of the entry contest to the professional teaching service may be useful to provide feedback on their training processes.

Key words: Teacher education - educational reform - public policy - educational models - principals

\section{Introducción}

El cambio en los sistemas educativos es un proceso que involucra a múltiples actores con diversos niveles de autoridad, que tienen ámbitos de negociación distintos y se mueven en muy diversos contextos. En este proceso de cambio existen varios modelos para diseñar una reforma educativa. Un modelo común es el top-down, en el cual las reformas se definen desde la estructura administrativa y política, es decir, de arriba hacia abajo. Este modelo mantiene el control central y suele ser unidireccional (Scott, Posner, Martin y Guzmán, 2015).

El modelo top-down está destinado al fracaso, a menos que instrumente algunos mecanismos para asegurar la participación de los actores en las distintas fases de su diseño e implementación. Considerar la participación de los interesados en la etapa de planeación de la reforma es importante en tanto que, de no ser así, los actores encontrarán en la fase de implementación su oportunidad para expresarse, generalmente en contra de la reforma o el cambio. Tal como sostiene Reimers (2011: 118) "cuando un sistema toca a múltiples intereses, el momento de la implementación es el momento en el cual se expresan aquellas voces que no fueron escuchadas en el momento de la consideración de opciones y de toma de decisiones".

De ahí que ningún proyecto de reforma, aun siendo top-down, pueda prescindir de la participación, en diferentes grados o momentos, de los actores educativos en la planeación o en el análisis técnico de las opciones políticas (Reimers, 2011). En el caso de la reforma educativa mexicana del sexenio pasado (2012-2018), la Secretaría de Educación Pública (SEP) presentó, en marzo de 2017, el Modelo Educativo para la Educación Obligatoria (SEP, 2017a) (en adelante Modelo Educativo 2017). Este modelo se elaboró a partir de los planteamientos recuperados en diversos foros nacionales, estatales e incluso escolares, mismos que fueron organizados con la finalidad de que la sociedad mexicana, en lo general, y grupos especializados, en lo particular, plantearan su opinión sobre un modelo educativo base y su propuesta curricular (PIPE-CIDE, 2016).

Este trabajo de investigación descriptivo y comparativo tuvo dos objetivos específicos: 1) Analizar las diferencias entre el apartado de formación inicial del capítulo "Formación y desa-

Diálo@os sobre Educación año 10 | número 19 | julio-diciembre 2019 | ISSN 2007-2171 
rrollo profesional docente" en el Modelo Educativo 2016 "Planteamiento pedagógico de la Reforma Educativa" (SEP, 2016a) y del mismo apartado en el Modelo Educativo 2017 (SEP, 2017a); y 2) Analizar la relación de los planteamientos de los participantes del "Foro de Consulta de Directivos de Escuelas Normales", celebrado el 17 de agosto de 2016, y el texto del apartado de Formación inicial del Modelo Educativo 2017 (SEP, 2017a).

\section{Los foros de consulta en la definición del Modelo Educativo}

El gobierno de Enrique Peña Nieto convocó a las fuerzas políticas del país a firmar el Pacto por México ${ }^{1}$ como su primera acción de gobierno, el 2 de diciembre de 2012. Los ejes rectores del pacto son: 1) El fortalecimiento del Estado mexicano; 2) La democratización de la economía y la política, así como la ampliación y aplicación eficaz de los derechos sociales; y 3) La participación de los ciudadanos como actores fundamentales en el diseño, la ejecución y la evaluación de las políticas públicas (Pacto por México, 2012).

La firma de este documento marca el inicio de la "Reforma educativa" que se instrumentó en el sexenio pasado en México. Este pacto establece la reforma legal y administrativa de la educación para incidir en tres propósitos: 1) aumentar la calidad de la educación básica, 2) aumentar la matrícula y asegurar la equidad, y 3) que el Estado mexicano "recupere la rectoría del sistema educativo nacional" (Pacto por México, 2012: 4). Quienes firmaron el Pacto por México fueron claros: la definieron como una reforma legal y administrativa en el sector educativo y establecieron nueve compromisos para este sector; sin embargo, ninguno de los cuales estuvo relacionado con definir un nuevo modelo educativo.

El planteamiento de la revisión del Modelo Educativo quedó consignado meses después en la Ley General de Educación (LGE), que en su artículo transitorio décimo segundo señala que "a efecto de dar cumplimiento a la obligación de garantizar la calidad en la educación, las autoridades educativas deberán proveer lo necesario para revisar el modelo educativo en su conjunto, los planes y programas, los materiales y métodos educativos" (DOF, 2013a: 64). Este compromiso se retomó en el Programa Sectorial de Educación, que señala en su línea de acción 1.3.10: "establecer mecanismos de consulta para revisar el modelo educativo en su conjunto para garantizar una educación de calidad" (DOF, 2013b: 8).

En cumplimiento de este compromiso, en 2014 la SEP organizó en distintas regiones del país los "Foros de Consulta Nacional para la Revisión del Modelo Educativo", organizados en tres ámbitos: educación básica, educación media superior y educación normal. Estos foros tuvieron cuatro objetivos principales: dar continuidad a la Reforma Educativa; analizar las necesidades actuales del país y del contexto mundial; llevar a cabo los ajustes necesarios para responder a

1 Firmado por el presidente de la república Enrique Peña Nieto y los presidentes de tres partidos políticos: Partido Acción Nacional (PAN), Partido Revoluionario Institucional (PRI) y Partido de la Revolución Democrática (PRD) (Pacto por México, 2012). 
las aspiraciones de los mexicanos; tomar en cuenta los rasgos y elementos específicos de cada región del país (SEP, 2014, párr. 6).

Dos años después, en julio de 2016, a partir de los planteamientos recabados en los foros de 2014, la SEP dio a conocer tres documentos que puso a consideración de todo el país: 1) Los fines de la educación en el siglo XXI (SEP, 2016b), 2) Modelo educativo 2016. Planteamiento pedagógico de la Reforma Educativa (2016a) y 3) La propuesta curricular para la educación obligatoria. Concretar un primer documento técnico del Modelo Educativo a tres años del inicio de la reforma, fue responsabilidad de dos secretarios de Educación, tres subsecretarios de Educación Básica y tres directores generales de Desarrollo Curricular.

La publicación de estos documentos dio inicio a un proceso de análisis al que se invitó a toda la sociedad mexicana mediante la realización de nuevos foros de consulta de muy diversa índole y ámbitos de alcance. Dicho proceso de análisis tuvo dos objetivos prioritarios: "promover un debate amplio y franco sobre los fines, los contenidos y los medios de la educación nacional para el siglo XXI" (PIPE-CIDE, 2016: 5) y "enriquecer la propuesta educativa presentada por la SEP con las opiniones y puntos de vista de los distintos segmentos de la sociedad mexicana" (PIPE-CIDE, 2016: 5).

En consonancia con el eje 3 del Pacto por México, que plantea la participación de los ciudadanos como actores fundamentales en el diseño, la ejecución y la evaluación de las políticas públicas, es la primera vez en la historia de la educación en México que una propuesta pedagógica estuvo en tantas mesas de análisis de tan diversa naturaleza. A partir de la publicación del Modelo Educativo 2016 (SEP, 2016a), se inició un proceso de consulta en distintos medios con el fin de recuperar las opiniones de los actores involucrados en educación obligatoria y mejorar la propuesta de Modelo Educativo presentada por la SEP.

Los principales medios para que los interesados compartieran sus opiniones fueron: $a$ ) foros organizados por la SEP, b) plataforma de consulta en línea, c) formatos en línea para los colectivos docentes, d) formatos en línea para Educación Media Superior, e) formularios en línea para recopilar opiniones de los participantes en los foros y encuentros, y $f$ ) documentos elaborados por diversas instituciones, entregados a la SEP (PIPE-CIDE, 2016).

La nueva edición de los foros de consulta sobre el Modelo Educativo fue organizada por la SEP del 20 de julio al 30 de septiembre de 2016. Con la finalidad de hacer una revisión desde diferentes perspectivas, se obtuvo la participación de distintos actores, organizaciones y organismos como: el Consejo Nacional de Participación Social en la Educación (CONAPASE), instituciones de educación superior, representantes de cámaras empresariales, académicos de educación media superior, académicos de educación básica, académicos y especialistas del sector educativo, directivos de escuelas normales, representantes del Sindicato Nacional de Trabajadores de la Educación (SNTE) y organizaciones de la sociedad civil, la Secretaría de Educación Pública y el Instituto Nacional para la Evaluación de la Educación. En total se realizaron 17 foros (PIPE-CIDE, 2016).

Diólo@os 
Los foros se organizaron en mesas de análisis, se contó con un moderador y un guion previamente definido para el desarrollo del diálogo. El guion solicitaba la opinión de los participantes sobre los cinco ejes del modelo. Cada mesa estuvo integrada por diez participantes, los cuales disponían de cinco minutos para expresar sus puntos de vista acerca de cada tema. Al finalizar cada conversación, se aplicó un cuestionario de opinión a los integrantes de las mesas sobre los fines de la educación en el siglo XXI, el perfil docente en la educación básica y en media superior, y la evaluación docente (PIPE-CIDE, 2016).

A partir de los resultados de esta segunda gran consulta pública, se relaboró el Modelo Educativo que fue publicado en marzo de 2017. El Modelo Educativo 2017 se estructuró en los mismos cinco ejes de la propuesta de 2016, si bien presentó cambios menores en su priorización y nomenclatura: 1) el planteamiento curricular, 2) la escuela al centro del sistema educativo, 3) formación y desarrollo profesional de los maestros, 4) inclusión y equidad, y 5) la gobernanza del sistema educativo (SEP, 2017a).

Este estudio analiza la manera en que las ideas expresadas en el "Foro de Consulta de Directivos de Escuelas Normales" (en adelante, Foro de directivos) de 2016 fueron recuperadas en la versión final del Modelo Educativo 2017 (SEP, 2017a), específicamente en lo que se refiere a la formación inicial de maestros, por considerar que esta es una etapa formativa fundamental para el logro de los fines de la educación del siglo XXI (SEP, 2016b).

\section{Metodología}

El método de análisis se dividió en dos fases: 1) Análisis de contenido de los documentos oficiales, y 2) Análisis de contenido de las opiniones vertidas en las mesas de trabajo del Foro de directivos. Los resultados de la primera fase fueron el insumo para desarrollar la segunda.

En ambas fases se utilizó como técnica el análisis de contenido, propuesta por Cáceres (2003). Esta técnica se ha definido de distintas formas, desde los enfoques que centran sus resultados en la cuantificación, hasta los que reconocen los datos cualitativos como el aspecto más valioso del procedimiento (Cáceres, 2003).

Si bien en el estudio se presentan datos cuantitativos, el interés se centra en el análisis cualitativo, es decir, la interpretación de los materiales para identificar relaciones entre estos. Cáceres (2003) presentó esta técnica como una alternativa para la relaboración, reducción y análisis de comunicaciones de manera comprensible, conservando el rigor y la sistematización con la que se concibió el material. Su propuesta se fundamenta en el modelo de Glaser y Strauss (1999, citados en Cáceres, 2003) y los planteamientos de Mayring (2000).

\section{Primera fase: Análisis de contenido de los documentos oficiales}

La primera etapa de la investigación fue el análisis de contenido de los documentos oficiales. Se seleccionó como universo el apartado de formación inicial de dos textos: el Modelo Educativo 
2016 (SEP, 2016a) y el Modelo Educativo 2017 (SEP, 2017a). Como unidades de análisis se seleccionaron fragmentos temáticos, es decir, aquellos conjuntos de palabras delimitadas a partir de sus significados (Cáceres, 2003). En la mayoría de los casos, las unidades de análisis correspondieron a un párrafo del texto; sin embargo, en algunas ocasiones se identificaron dos unidades en un párrafo. Se realizó un proceso de codificación abierta.

Primero se efectuó una lectura del apartado de Formación inicial del Modelo Educativo 2016 y se identificaron los temas en cada unidad de análisis. Posteriormente, se leyó el texto homónimo del Modelo Educativo 2017 y se realizó el mismo proceso. En los casos en que se identificaron fragmentos de texto iguales o que compartían características comunes con alguna de las unidades de análisis del Modelo Educativo 2016, se asignó el mismo código (Strauss y Corbin, 2002). Los códigos pasaron a ser categorías textuales, definidas a partir de la idea principal de las unidades de análisis en cada código (Cáceres, 2003). Se obtuvieron como resultado 19 categorías temáticas, mismas que corresponden a las ideas principales de las unidades de análisis, como se indicó en el párrafo anterior. Se realizó un inventario de las categorías identificadas en cada uno de los textos analizados (SEP, 2016a y 2017a), con la finalidad de tener claridad respecto a las ideas que estaban presentes en ambos modelos y las que solamente se presentaron en uno de los dos documentos.

Como producto final de esta fase, se obtuvo una tabla que en la primera columna presentaba las 19 categorías, mientras que en la segunda y tercera se indicó con una " $X$ " si la categoría estaba presente en el Modelo Educativo 2016 y el Modelo Educativo 2017, respectivamente.

\section{Segunda fase: Análisis de contenido de los Foros de Directivos de Escuelas Normales}

Esta fase tuvo como objetivo analizar la relación de los planteamientos de los participantes en el Foro de Directivos de Escuelas Normales con el texto del Modelo Educativo 2017. De todos los foros realizados, se seleccionó el de directivos de escuelas normales en tanto que se consideró que podría ser el foro en el que se hicieran observaciones más fundamentadas respecto de la formación inicial de maestros en México.

En este caso, el preanálisis consistió en la identificación de los videos de las mesas de trabajo del foro, la descripción de los participantes, selección de los fragmentos de video y la transcripción de los mismos, como se describe a continuación. El Foro de directivos se realizó el día 17 de agosto de 2016 y estuvo organizado en diez mesas de trabajo. De la presentación de los integrantes de las mesas de trabajo se identificaron las siguientes características: cargo, institución y entidad federativa de procedencia. Dado que el interés de la investigación está ubicado en la formación inicial de los docentes de educación básica, se identificaron los fragmentos de video en los que los coordinadores plantearon el tema de la formación inicial en la sección del tema "Formación y desarrollo profesional docente". Finalmente, se transcribieron los fragmentos de videos seleccionados. Se transcribieron aproximadamente tres horas y media de

Diólo pos sobre Educación año 10 | número 19 | julio-diciembre 2019 | ISSN 2007-2171 
grabación, con un resultado de 48 páginas en procesador de textos (25,409 palabras). Una vez identificados los fragmentos, se colocó una etiqueta para identificar la mesa del foro a la que correspondía el texto y la página de la transcripción en la que se encontraba.

Primero se hizo una categorización deductiva. Dado que en esta fase el objetivo era analizar la relación de los planteamientos de los participantes en el foro de directivos con el texto del Modelo Educativo 2017, se utilizaron las categorías identificadas en la fase uno. Posteriormente, se hizo una codificación y categorización inductiva. En este paso se trabajó con las unidades de análisis que no pudieron ser ubicadas en las categorías ya establecidas. En primer lugar, se identificó el tema al que se refería cada una de las categorías y se asignó un código; en segundo, se agruparon las unidades que correspondían al mismo código; en tercero, se realizó una revisión para asegurar que estos fueran excluyentes; $y$, por último, se establecieron la categorías tomando como referencia los códigos.

Al realizar el análisis de la relación entre los planteamientos de los participantes en el Foro de directivos y las categorías derivadas de las ideas principales en el Modelo Educativo 2016 y el Modelo Educativo 2017, se encontró que los resultados podían organizarse en tres casos, los cuales se describen en la tabla 1.

Tabla 1 Descripción de casos identificados

\begin{tabular}{|c|l|l|}
\hline Caso & \multicolumn{1}{|c|}{ Definición } & \multicolumn{1}{|c|}{ Descripción } \\
\hline 1 & $\begin{array}{l}\text { Planteamientos similares (presentes) } \\
\text { en el Foro de directivos de Escuelas } \\
\text { Normales y el Modelo Educativo 2017 } \\
(\mathrm{F}=\mathrm{M} 17)\end{array}$ & $\begin{array}{l}\text { En este grupo se ubicaron las ideas expresadas } \\
\text { en el Foro de directivos de Escuelas Normales } \\
\text { y que se ven reflejadas en el Modelo Educativo } \\
2017 \text { (SEP, 2017). }\end{array}$ \\
\hline 2 & $\begin{array}{l}\text { Ideas presentes en los modelos que } \\
\text { no fueron mencionadas en el Foro de } \\
\text { directivos de Escuelas Normales } \\
(\mathrm{M} 16,17 \neq \mathrm{F})\end{array}$ & $\begin{array}{l}\text { Se refiere al análisis de los planteamientos de los } \\
\text { documentos oficiales que no se identificaron en } \\
\text { los comentarios de los participantes del Foro de } \\
\text { directivos de Escuelas Normales }\end{array}$ \\
\hline 3 & $\begin{array}{l}\text { Planteamientos del Foro de directivos } \\
\text { de Escuelas Normales no identifica- } \\
\text { dos (ausentes) en el modelo }(\mathrm{F} \neq \mathrm{M} 17)\end{array}$ & $\begin{array}{l}\text { Se refiere a los planteamientos de los participan- } \\
\text { tes, considerados relevantes, que no se encontra- } \\
\text { ron reflejados en el documento Modelo Educati- } \\
\text { vo 2017 (SEP, 2017a). }\end{array}$ \\
\hline
\end{tabular}

Nota: Códigos utilizados: M16= Modelo educativo 2016, M17= Modelo educativo 2017 y F= Foro de Directivos de Escuelas Normales.

Fuente: Elaboración propia con los datos del Foro de Directivos de Escuelas Normales y SEP (2017a). 


\section{Resultados}

A continuación se presentan los resultados del estudio por cada una de las fases descritas.

\section{Primera fase: Análisis de contenido de los documentos oficiales}

A partir del análisis de los documentos oficiales, se obtuvieron como resultado 19 categorías temáticas, mismas que corresponden a las ideas principales de ambas versiones del Modelo Educativo (SEP, 2016a y 2017a).

En la tabla 2 se presentan las similitudes y diferencias identificadas en las ideas principales de las dos versiones del Modelo Educativo. En la primera columna se exponen las categorías, mientras que en la segunda y tercera columna se especifica con una " $X$ " si la categoría se encontró en el Modelo Educativo 2016, en el de 2017, o en ambos.

Tabla 2 Similitudes y diferencias identificadas en las ideasprincipales

de las dos versiones del Modelo Educativo

\section{Categorías \\ Ideas principales en ambas versiones del Modelo Educativo \\ El sector se abre a profesionistas con vocación docente, pero con una formación}

2016 universitaria diversa (SEP, 2017a, p. 140).

X $\quad X$

Es indispensable que las escuelas normales impulsen los cambios necesarios para actualizarse y seguir siendo el pilar de la formación inicial de los docentes de educación básica (SEP, 2016a, p. 60 y 2017, p. 141).

Es preciso actualizar las licenciaturas en educación que ofrecen las escuelas nor- $\quad X \quad X$ males (SEP, 2017a, p. 141).

Las escuelas normales tienen que organizarse como instituciones de educación superior y funcionar con la calidad necesaria para ofrecer opciones de innovación $\quad X \quad X$ (SEP, 2017a, p. 144).

Es importante fortalecer las relaciones de colaboración entre las normales y otras instituciones de educación superior para robustecer la formación docente y la construcción del conocimiento (SEP, 2017a, p. 146). [Movilidad docente y estudiantil, en la misma entidad, en el nivel nacional e internacional].

\begin{tabular}{l}
\hline $\begin{array}{l}\text { La transformación de las normales debe considerar, desde luego, la renovación y } \\
\text { fortalecimiento de la planta docente (SEP, 2016a, p. 60). }\end{array}$ \\
\hline $\begin{array}{l}\text { La formación inicial de los docentes es tan importante como la formación continua } \\
\text { (SEP, 2017a, p. 140). }\end{array}$ \\
$\begin{array}{l}\text { La profesión docente debe ser una opción atractiva para los buenos egresados de } \\
\text { bachillerato (SEP, 2017a, p. 141). }\end{array}$ \\
$\begin{array}{l}\text { Las escuelas normales requieren y merecen los apoyos necesarios (SEP, 2017a, p. } \\
\text { 141). }\end{array}$ \\
\hline
\end{tabular}




\begin{tabular}{ll}
\hline $\begin{array}{l}\text { Es preciso actualizar [...] los planes y programas de estudios afines de otras institu- } \\
\text { ciones de educación superior (SEP, 2017a, p. 141). }\end{array}$ & $X$ \\
\hline $\begin{array}{l}\text { Los maestros deben desarrollar el dominio sobre los contenidos de los aprendiza- } \\
\text { jes clave, así como de las estrategias para transmitirlos (SEP, 2017a, p. 142). }\end{array}$ & $X$ \\
\hline $\begin{array}{l}\text { La formación inicial debe equipar a los maestros con las bases pedagógicas y di- } \\
\text { dácticas que les permitan poner en práctica los principios pedagógicos en que se } \\
\text { sustenta el modelo educativo (SEP, 2017a, p. 142). }\end{array}$ & $X$ \\
\hline $\begin{array}{l}\text { La formación inicial debe desarrollar en los maestros la capacidad para aprender a } \\
\text { aprender (SEP, 2017a, p. 142). }\end{array}$ & $X$ \\
\hline $\begin{array}{l}\text { Es fundamental que todos los maestros -no solamente quienes enseñan esta } \\
\text { asignatura- dominen este idioma [inglés]. (SEP, 2017a, p. 143) }\end{array}$ & $X$ \\
\hline $\begin{array}{l}\text { Es clave que las escuelas normales, al igual que las demás instituciones de educa- } \\
\text { ción superior, tengan acceso a las nuevas tecnologías para formar a los docentes } \\
\text { en estas competencias (SEP, 2017a, p. 143). }\end{array}$ & $X$ \\
\hline $\begin{array}{l}\text { El modelo educativo plantea la inclusión y la equidad como principios básicos } \\
\text { transversales, lo cual exige que la formación inicial adopte una perspectiva equita- } \\
\text { tiva, inclusiva e intercultural (SEP, 2017a, p. 143). }\end{array}$ & $X$ \\
\hline $\begin{array}{l}\text { En las zonas donde predomine la población indígena es preciso ofrecer programas } \\
\text { de formación inicial en preescolar y primaria intercultural bilingüe que promuevan de } \\
\text { manera particular el conocimiento de la lengua en que esos futuros maestros previ- } \\
\text { siblemente han de enseñar (SEP, 2017a, p. 144). }\end{array}$ & $X$ \\
\hline $\begin{array}{l}\text { Es necesario diseñar un sistema de incentivos que promueva la excelencia en las } \\
\text { escuelas normales y fomente los cambios institucionales (SEP, 2017a, p. 144). }\end{array}$ & $X$ \\
\hline $\begin{array}{l}\text { Las escuelas normales deben realizar un esfuerzo interno por acreditar la calidad } \\
\text { de los servicios que ofrecen (SEP, 2017a, p. 145). }\end{array}$ \\
\hline
\end{tabular}

Fuente: Elaboración propia a partir de SEP (2016a y 2017a).

Como se puede observar en la tabla 2, se identificaron cinco ideas comunes en ambos planteamientos del Modelo Educativo. Asimismo, se reconocieron 14 diferencias, una categoría presente en el Modelo Educativo 2016 que no fue recuperada en la versión de 2017 y 13 categorías encontradas únicamente en el replanteamiento del Modelo Educativo 2017.

De los 13 planteamientos que únicamente se encontraron en el Modelo Educativo 2017, nueve categorías se refieren a la visión o ideales de la formación inicial $(7,8,11,12,13,14,15$, 16 y 17); tres a los apoyos que las escuelas normales requieren para su fortalecimiento $(9,18$ y 19); y una idea más está destinada a la actualización de planes de estudio afines al normalismo dado el hecho de que, a partir de 2016, la Ley General del Servicio Profesional Docente permite el ingreso a egresados de licenciaturas afines como docentes de educación preescolar y primaria (Cordero y Salmerón, 2017).

En el Modelo Educativo 2017, la idea que no se recuperó es “La transformación de las normales debe considerar, desde luego, la renovación y fortalecimiento de la planta docente" (SEP, 
2016a, p. 60). El concepto de renovación de la planta docente no queda claro en el texto del Modelo Educativo 2016. No se explicita en el texto lo que implica esta renovación, el tema no se abordó en las mesas de trabajo y tampoco fue incluido en el Modelo Educativo 2017 (SEP, 2017b).

La única referencia que se hace a los maestros de las escuelas normales en el Modelo 2017 es para señalar que hacen falta "maestros preparados" (SEP, 2017a: 141) para tener instituciones "sólidas". En las mesas de trabajo se encontró que la noción de fortalecimiento está asociada a la mejora continua, actualización y profesionalización de los maestros de las escuelas normales de acuerdo al Modelo Educativo. Los participantes de las mesas de trabajo formularon los siguientes comentarios al respecto:

El punto clave para fortalecer las escuelas normales es que haya procesos de selección y apoyo para la profesionalización, pero necesitamos tener de entrada profesores capacitados (M3-06). ${ }^{2}$

Es bueno, es conveniente que ese sea el enfoque, pero, sí se requiere de una preparación para que los maestros que trabajan en las Escuelas Normales se puedan actualizar y al mismo tiempo puedan impulsar el trabajo que se necesita de formación para sus propios alumnos (M8-32).

Entonces ¿hasta dónde estamos dispuestos? Aquí están los directores de las Escuelas Normales, aquí estamos las autoridades. ¿Hasta dónde, realmente? Ya lo dijeron ustedes muy bien, en otros países se forma a los directivos. Tendríamos que empezar a formarnos nosotros mismos para poder estar en condiciones y cumplir con las expectativas de este nuevo modelo (M9-39).

En el entendido de que "la renovación y fortalecimiento de la planta docente" se refiere a la formación continua, actualización y profesionalización de los catedráticos normalistas, los directivos sí consideraron esta idea como un punto clave que requiere ser atendido en sus instituciones, como una de las vías que les permiten mejorar en cuanto a la calidad del servicio que ofrecen.

\section{Segunda Fase: Análisis de contenido de los Foros de Directivos de Escuelas Normales - Identificación de participantes}

En las diez mesas de trabajo se contó con la participación de 122 integrantes. ${ }^{3}$ Como se indica en el nombre del foro, este estuvo integrado por directivos de escuelas normales de las distintas entidades del país. En la tabla 3 se presenta la distribución de los participantes de acuerdo a su cargo.

2 Código de transcripción: (Número de mesa de trabajo-página del documento transcito). Ejemplo: Comentario en mesa de trabajo 2, ubicado en página 10 (M2-10)

3 El informe del PIPE-CIDE (2016) reporta asistencia de 125 personas.

Diólo pos 
Tabla 3 Distribución de los participantes por cargo

\begin{tabular}{|l|r|r|}
\hline Participantes & n & \% \\
\hline Directores y subdirectores & 58 & 48 \\
\hline Directores estatales & 36 & 30 \\
\hline Representantes del sindicato & 3 & 2 \\
\hline Representantes de la DGESPE & 11 & 9 \\
\hline Representantes del CIDE & 10 & 8 \\
\hline Sin especificar & 4 & 3 \\
\hline Total & 122 & 100 \\
\hline
\end{tabular}

Fuente: Elaboración propia.

Se registró participación de los 32 estados de la república mexicana. Puebla fue el estado que tuvo mayor representación, con cinco integrantes, mientras que Zacatecas únicamente contó con la presencia de un representante.

Asimismo, estuvieron presentes representantes de los distintos tipos de normales, entre las cuales se encuentran: Escuela Normal, Normal Superior, Normal Experimental, Normal Rural, Normal Urbana y Escuelas de Educación Física.

- Análisis de la relación entre las temáticas del Foro de directivos de Escuelas Normales

y las ideas principales de ambas versiones del Modelo Educativo

A partir de las 19 categorías identificadas en la fase 1 de este estudio, se analizaron las transcripciones del Foro de directivos, a fin de reconocer si las ideas planteadas en las mesas de trabajo fueron recuperadas en el Modelo Educativo 2017. Como resultado se encontraron tres casos distintos, los cuales se describen a continuación.

\section{Caso 1. Planteamientos del Foro de directivos de Escuelas Normales presentes en el Modelo Educativo 2017}

Catorce de las 19 categorías identificadas en la fase 1 -expresadas en el Modelo Educativo 2017 (SEP, 2017a) — fueron mencionadas por los participantes de las distintas mesas de análisis. Los temas abordados por los participantes de las mesas de trabajo en el Foro para directivos se listan en la tabla 4, ordenados de acuerdo a la cantidad de unidades de análisis relacionados a cada categoría. 
Tabla 4 Número de unidades de análisis en cada categoría

\begin{tabular}{|c|c|}
\hline Categoría & $\begin{array}{l}\text { No. de unidades } \\
\text { de análisis }\end{array}$ \\
\hline $\begin{array}{l}\text { Es preciso actualizar las licenciaturas en educación que ofrecen las escuelas } \\
\text { normales }\end{array}$ & 20 \\
\hline $\begin{array}{l}\text { Las escuelas normales tienen que organizarse como instituciones de educa- } \\
\text { ción superior y funcionar con la calidad necesaria para ofrecer opciones de } \\
\text { innovación. }\end{array}$ & 10 \\
\hline Las escuelas normales requieren y merecen los apoyos necesarios. & 7 \\
\hline $\begin{array}{l}\text { Es importante fortalecer las relaciones de colaboración entre las normales y } \\
\text { otras instituciones de educación superior para robustecer la formación do- } \\
\text { cente y la construcción del conocimiento [Movilidad docente y estudiantil, en } \\
\text { la misma entidad, a nivel nacional e internacional]. }\end{array}$ & 7 \\
\hline $\begin{array}{l}\text { Es indispensable que las escuelas normales impulsen los cambios necesarios } \\
\text { para actualizarse y seguir siendo el pilar de la formación inicial de los docen- } \\
\text { tes de educación básica. }\end{array}$ & 7 \\
\hline $\begin{array}{l}\text { Las escuelas normales deben realizar un esfuerzo interno por acreditar la } \\
\text { calidad de los servicios que ofrecen. }\end{array}$ & 3 \\
\hline $\begin{array}{l}\text { El sector se abre a profesionistas con vocación docente, pero con una forma- } \\
\text { ción universitaria diversa. }\end{array}$ & 3 \\
\hline $\begin{array}{l}\text { Es fundamental que todos los maestros —no solamente quienes enseñan } \\
\text { esta asignatura_-dominen este idioma [inglés] }\end{array}$ & 3 \\
\hline $\begin{array}{l}\text { La formación inicial debe equipar a los maestros con las bases pedagógicas y } \\
\text { didácticas que les permitan poner en práctica los principios pedagógicos en } \\
\text { que se sustenta el modelo educativo. }\end{array}$ & 2 \\
\hline $\begin{array}{l}\text { La profesión docente debe ser una opción atractiva para los buenos egresa- } \\
\text { dos de bachillerato. }\end{array}$ & 2 \\
\hline $\begin{array}{l}\text { Es clave que las escuelas normales, al igual que las demás instituciones de } \\
\text { educación superior, tengan acceso a las nuevas tecnologías para formar a los } \\
\text { docentes estas competencias. }\end{array}$ & 2 \\
\hline $\begin{array}{l}\text { Es preciso actualizar [...] los planes y programas de estudios afines de otras } \\
\text { instituciones de educación superior. }\end{array}$ & 1 \\
\hline $\begin{array}{l}\text { La formación inicial debe desarrollar en los maestros la capacidad para } \\
\text { aprender a aprender. }\end{array}$ & 1 \\
\hline $\begin{array}{l}\text { En las zonas donde predomine la población indígena es preciso ofrecer pro- } \\
\text { gramas de formación inicial en preescolar y primaria intercultural bilingüe } \\
\text { que promuevan de manera particular el conocimiento de la lengua en que } \\
\text { esos futuros maestros previsiblemente han de enseñar. }\end{array}$ & 1 \\
\hline
\end{tabular}

Fuente: Elaboración propia. 
Como se observa en la tabla 4, la unidad de análisis más recurrente, mencionada por los participantes, fue la actualización de las licenciaturas en educación que ofrecen las escuelas normales (SEP, 2017a). Al respecto, uno de los participantes precisó:

\begin{abstract}
Yo lo que creo también es que estamos, en este momento, ante una oportunidad inmejorable para que el nuevo planteamiento curricular de las Escuelas Normales sea pertinente. Me refiero a que si estos documentos curriculares o la propuesta curricular para la Educación Básica, perdón, obligatoria, que se va a aplicar a partir del 2018, tengo entendido, quiere decir que tenemos un tiempo muy razonable como para que, paralelamente, las Escuelas Normales empiecen a transformar sus planes y programas de estudio. Es inmejorable esta oportunidad porque por primera vez en la historia de la educación en México, se presentaría una propuesta curricular para Educación Básica y Educación Media Superior a la par que la de la formación inicial de los nuevos maestros. Es un tiempo muy razonable para hacerlo (M9-40).
\end{abstract}

De manera particular, se recuperaron unidades de análisis en esta categoría que reflejan el interés de los directivos de escuelas normales en alinear los planes y programas de estudios de las licenciaturas que ofrecen, con el planteamiento pedagógico del nuevo Modelo Educativo, idea que está plasmada en ambos documentos del modelo. Relativo a ello, algunos de los comentarios fueron:

Mucho tenemos por hacer las escuelas normales, desde el diseño curricular para que los estudiantes que salen de la escuela normal salgan con las competencias que requieren para cumplir con lo que se dice en este modelo educativo (M1-02).

Pero es importante que esta formación inicial incluya principalmente lo que se está planteando en este nuevo modelo educativo. Digamos, hablan de un maestro... que las escuelas tengan maestros de inglés, por ejemplo (M10-43).

La siguiente idea que tuvo mayor frecuencia fue la necesidad de que las escuelas normales se organicen como instituciones de educación superior y funcionen con la calidad necesaria para ofrecer opciones de innovación (SEP, 2017a). Al respecto, uno de los participantes comentó:

Es importante resaltar la gran necesidad que se tiene de formación e integración de cuerpos académicos, es fundamental para tener formación de a de veras pues hay que generar cuerpos académicos. No hay que tenerle miedo a ese tema. Yo creo que esa va hacer notablemente la diferencia, nos va hacer verdaderamente competitivos ahora que también van a participar las universidades en tema de la formación. Entonces es un asunto que necesitamos desarrollar urgente, necesitamos desarrollar investigación de campo, desarrollar investigación en lo estrictamente práctico y propio de la formación normal, pero a veces parece incongruente la necesidad por un lado y por el otro el freno porque en alguna o 
muchas ocasiones el ser parte de un cuerpo académico te implica, va en contra de tus horas clases. Entonces las escuelas no apoyan estas iniciativas. Entonces sí es importante mencionar que ante esta gran necesidad de los cuerpos académicos, se deba también contar con la colaboración de las escuelas para que se permita integrar a los docentes que participen en los cuerpos académicos y se les deje participar, se les den las facilidades y todo el apoyo para poderlo hacer porque eso es lo que va hacer la diferencia afuera con respecto a los demás, la educación de calidad ya no tiene reversa, ya no tiene marcha atrás (M5-17).

Las declaraciones anteriores fundamentan la pertinencia de los temas expuestos en las mesas de análisis y reflejan las ideas que los participantes consideran relevantes como acciones para fortalecer las escuelas normales y la profesionalización de su planta docente, lo que a su vez repercute en la mejora de la formación inicial de los estudiantes normalistas.

\section{Caso 2: Planteamientos del Modelo Educativo 2017 no mencionados (ausentes) en el Foro de Directivos de Escuelas Normales}

Se encontraron ideas en el Modelo Educativo 2017 que no fueron planteadas de manera explícita en el Foro de directivos. Este es el caso de las siguientes cuatro categorías:

- La formación inicial de los docentes es tan importante como la formación continua.

- Los maestros deben desarrollar el dominio sobre los contenidos de los aprendizajes clave, así como de las estrategias para trasmitirlos.

- El modelo educativo plantea la inclusión y la equidad como principios básicos transversales, lo cual exige que la formación inicial adopte una perspectiva equitativa, inclusiva e intercultural.

- Es necesario diseñar un sistema de incentivos que promueva la excelencia en las escuelas normales y fomente los cambios institucionales.

Es posible que estas ideas se hubieran planteado en foros realizados con otros actores que no fueron estudiados en este trabajo. Es interesante destacar en este conjunto de ideas el compromiso que adquiere la autoridad federal para definir un sistema de incentivos relacionado con la "excelencia académica", sin precisar o definir lo que se entiende por este concepto.

\section{Caso 3: Planteamientos del Foro de Directivos de Escuelas Normales}

\section{no identificados (ausentes) en el Modelo Educativo 2017}

Por último, en este caso se ubican dos ideas que se abordaron en las distintas mesas de trabajo del Foro de Directivos, que no se encuentran reflejadas en el apartado de "Formación Inicial del Modelo Educativo 2017" (SEP, 2017a). Se consideró importante mencionar estas ausencias

Diálo@os

sobre Educación

año 10 | número 19 | julio-diciembre 2019 | ISSN 2007-2171 
en el presente análisis ya que ideas fueron referidas por varios actores en distintos momentos, y retomadas por los participantes siguientes como temas de interés. Estas ideas fueron: 1) las escuelas normales pueden tener un nuevo campo de acción en la formación de docentes en servicio, y 2) los resultados de la evaluación del ingreso al Servicio Profesional Docente (SPD) deberían ser un insumo para la mejora de los procesos formativos de las escuelas normales.

\section{1) Las escuelas normales pueden tener un nuevo campo de acción en la formación de docentes en servicio}

La primera idea mencionada por los directivos de las escuelas normales se refiere a la posibilidad de tener un liderazgo y responsabilidad en la formación continua de los maestros en servicio, tanto para los egresados de sus escuelas como para los docentes que tienen otro título universitario ajeno a las normales.

Al ser instituciones de educación superior no podemos limitarnos con la formación inicial [...] también necesitamos la oferta de formación continua para que retroalimenten el trabajo que día a día hacemos. [...] No nos debe de asustar que egresados de universidades van a competir por plazas docentes, al contrario, como escuelas normales tenemos que responder a las necesidades en cuanto a formación de nivel medio superior, necesitamos responder con programas pertinentes [...] que les den ese respaldo pedagógico que no tienen (M1-4).

También otro punto que habla de la actualización de conocimientos y competencias de los docentes es que las instituciones formadoras de docentes sean reivindicadas como espacios de aprendizaje y desarrollo profesional docente para la estrategia de formación continua en diversas modalidades (M3-5). Creo que la visión tendría que ser que la formación docente es un continuo que inicia cuando el chico entra al primer semestre de su escuela normal y termina cuando se jubila. Y las escuelas normales tendríamos que estar comprometidas en todo ese trayecto (M6-21).

Me asalta la duda, la preocupación. Un contador público, un abogado y, con todo respeto para todos los profesionistas, una licenciada en Comunicación, etcétera ¿qué hará en un grupo, en una escuela multigrado unitaria o bidocente? Es necesario, es urgente, que desde las escuelas normales tengamos esas posibilidades de poder dar esta preparación alterna o capacitación o formación para que esos maestros idóneos, que no cuentan con el perfil normalista, como nosotros tenemos o los alumnos que egresan de nuestra institución formadora de docentes, puedan tener esas herramientas y poder así, lograr los fines de la educación que en este nuevo modelo educativo de plantea (M7-28-29).

Se mencionó que al observar la disminución que se ha presentado en la matrícula de ingreso en las escuelas normales, se hace necesario diseñar nuevas acciones que permitan a las normales continuar con sus labores: 
Nosotros lo estamos viendo, como comentaba el compañero, en la baja matrícula de ingreso, eso también, se tienen que mover ciertos elementos. Empezar a incursionar, lo han hecho otras normales, no quiere decir que no se ha hecho, en los diplomados, cursos, maestrías, doctorados, pero más enfocados a estos. Tratar de tener la formación continua de nuestros egresados, darles seguimiento, quiénes no tuvieron buenos resultados en la evaluación diagnóstica y estarlos rectificando para poderles dar esa necesidad de formación continua que tienen, esa sería la propuesta (M5-16).

Se indicó la posibilidad de que las escuelas normales brinden tutoría a los docentes no idóneos, modalidad introducida en la Ley General del Servicio Profesional Docente (DOF, 2013c) para la formación de maestros en servicios, en concreto expresó:

[...] nos convertiríamos en otra cosa maravillosa, en los tutores de los maestros que no han logrado el nivel, porque para que yo sea guía necesito ser un experto, entonces creo que es una gran oportunidad para nosotros los maestros de las instituciones formadoras de docentes de que estemos a la vanguardia en cuanto a los programas de estudio y podamos ser nosotros los tutores (M5-15).

Estas ideas no se mencionan en el apartado de "Formación Inicial del Modelo Educativo 2017". En el capítulo de "Formación Continua para Docentes" se plantea que "en los procesos de formación docente se debe impulsar la participación de las instituciones de educación superior y las escuelas normales" (SEP, 2017a: 135). Sin embargo, no se reconoce en esta afirmación el énfasis que los directivos hacen acerca de tener un liderazgo claro en esta materia.

\section{2) Los resultados de la evaluación del ingreso al SPD deberían ser un insumo para la mejora de los procesos formativos de las escuelas normales}

La segunda idea que plantearon los participantes del foro, y que no fue retomada en el Modelo Educativo 2017, es la importancia de que los resultados de las evaluaciones del SPD sean un insumo para la mejora de los procesos formativos que se llevan a cabo en las escuelas normales. Es decir, que los resultados que obtienen los egresados normalistas en los concursos de oposición para el ingreso al servicio tengan un sentido formativo y sean de utilidad para las autoridades y docentes de las escuelas normales.

Al respecto, los directores de escuelas normales opinaron que:

[...] estas evaluaciones, considerando que el INEE ${ }^{4}$ es un órgano independiente sí es cierto, pero los resultados tendrían que servirnos para reformular y replantear, o sea, que no solamente sea el $50 \%$ resultó idóneo y no sabemos en qué sentido, específicamente en qué rubro tenemos que mejorar, yo creo que si la evaluación la consideramos formativas tendría que tener ese sentido de informar qué estamos haciendo bien, qué tenemos que replantear y, finalmente, para crecer (M1-4).

4 Instituto Nacional para la Evaluación de la Educación

Diálo@os 
Mi propuesta va en el sentido de los resultados de evaluación. Toda evaluación con consecuencias es una evaluación verdadera [...] las que hace el INEE con todas las cuestiones científicas, se deben de calibrar para que podamos acercarnos a la pertinencia y esos resultados nosotros no solamente los deberíamos de utilizar para las cuestiones de orden laboral, sino también como un insumo para la evaluación curricular y que nos permita ahora sí en flexibilidad, adaptar el currículo para que ahora sí haya un egresado de las normales en mejores condiciones (M3-5-6).

En este sentido, sabemos que el Servicio Profesional Docente está enfocado en todos estos procesos: ingreso, promoción, reconocimiento y permanencia de los docentes [...] Ahí la propuesta podría ser que estos resultados de las evaluaciones pudieran ser un referente formal para la transformación curricular que tanto hemos mencionado en las instituciones formadoras de docentes, dado que, históricamente, se han diseñado e implementado planes de estudio que han sido evaluados en este referente (M10-45).

Esta demanda plantea que la manera en que se están entregando los resultados de la evaluación de ingreso no responde a las necesidades de los directivos de las normales para retroalimentar a profesores y alumnos en cuanto a las áreas de oportunidad que pudieran presentar, con el fin de buscar estrategias para fortalecer a los profesionales de la educación que así lo requieran, de acuerdo con los reportes de resultados del SPD.

\section{Discusión y conclusiones}

Generar un nuevo modelo educativo no fue un compromiso original de la reforma educativa. Posiblemente fue incorporado en la Ley General de Educación a partir de las críticas que la reforma administrativa y legal recibió por parte de los actores educativos y del mundo académico en su momento de definición (Ornelas, 2013; Zebadúa, 2013).

Durante cuatro años sucedió una serie de acciones que culminaron en la definición de un nuevo modelo educativo: dos etapas de foros nacionales y la publicación de un documento que sirvió de base para la discusión. En este proceso top-down, la SEP impulsó mecanismos de participación ciudadana para que la sociedad expresara sus opiniones acerca del tema educativo por ser de interés público, ya que, "la consulta pública, como mecanismo para enriquecer el modelo educativo también responde al esfuerzo de las autoridades por ganar legitimidad" (Abellán, 2016: 78). Dada la complejidad de la sociedad actual, dicha participación se dio de manera representativa (Proyecto Estado de la Nación, 2001, IEPC, s.f.; PIPE-CIDE, 2016) y a través de diferentes acciones.

La participación en las mesas de los directivos tuvo los límites asignados a la dinámica de trabajo, por tiempo y orden de presentación de los temas. También tuvo los límites propios del tipo de ejercicio al que fueron convocados. No se trataba de opinar sobre un modelo específico en concreto o sobre un plan específico, sino sobre una serie de ideas deseables para la formación inicial de maestros. De ahí que la opinión de los directivos también se moviera en el plano 
del deber ser, a partir de sus propias experiencias. Tal como plantea Reimers, estos ejercicios tienen un valor muy específico:

Bajo el nombre de "planificación participativa" se llevan a cabo prácticas diversas, muchas de las cuales consisten en ejercicios de participación y movilización social, en las que no es fácil reconocer que se busque maximizar un conjunto de objetivos en los esfuerzos de reformar un sistema educativo. Las interminables listas de deseos generadas en muchas de estas consultas maximizan el objetivo de movilizar políticamente; pero no es claro que maximizan el aprovechamiento de los recursos disponibles, las oportunidades de aprendizaje, la equidad en el sistema, la calidad o pertinencia de lo que se enseña. En consecuencia, muchos de estos ejercicios son más participación, que planificación (Reimers, 2011:119).

Algunos de los planteamientos de los directivos de escuelas normales expresados en las mesas de trabajo, se han planteado desde hace décadas. Por ejemplo, Figueroa (2000: 120), hace casi 20 años, mencionó que el normalismo enfrenta retos y que el panorama "no es muy halagador", enuncia problemas como el descenso de matrícula, desfase de lo que se enseña en las aulas de las escuelas normales y lo que se necesita implementar en el campo laboral, aislamiento de las normales y falta de reconocimiento como IES. Estos temas han permanecido en la agenda educativa por un largo periodo y no han sido atendidos.

Uno de los temas relevantes en las mesas de trabajo fue la necesidad de fortalecer la planta académica de las instituciones formadoras de docentes, para incrementar su profesionalización mediante la formación, actualización y la implementación de procesos de selección. De esta manera, los catedráticos normalistas contarán con las competencias requeridas para formar a los futuros profesionales de la educación.

En concreto, destacan dos ideas importantes que no fueron incorporadas en el Modelo Educativo 2017. Una de ellas tiene que ver con definir acciones que fortalezcan a la institución normalista considerando su participación en tareas que tradicionalmente han sido de la competencia del área de formación continua, tales como apoyar la formación de posgrado de sus egresados o atender las tareas formativas que plantea la propia Ley General de Servicio Profesional Docente para los distintos actores educativos (DOF, 2013c).

La otra acción importante que plantean los directivos enfáticamente es la posibilidad de que los resultados del concurso de ingreso al Servicio Profesional Docente puedan ser de utilidad para retroalimentar sus procesos formativos. De esta forma, subrayan la falta de articulación de los procesos evaluativos y formativos ya que, aunque esta es una promesa de la reforma, no ha podido concretarse.

Si bien el apartado de "Formación inicial" del documento del Modelo Educativo 2017 (SEP, 2017a) presenta un mayor número de ideas y están mejor desarrolladas que en el texto anterior, este Modelo Educativo 2017 no llega a constituirse como un modelo para la formación inicial 
de docentes. El documento incluye y considera esta etapa de la formación docente dada la importancia de hacer consecuentes los planteamientos del modelo para la educación obligatoria con la formación inicial de profesores; como menciona Pérez (2015), los cambios aplicables a la educación básica tienen inciden en la formación inicial de los docentes. Los planteamientos que se incluyen en el texto (SEP, 2017a) son un conjunto de ideas que pueden ayudar a definir un modelo para la formación inicial y donde se reconocen tanto las ideas originales del Modelo Educativo 2016 (SEP, 2016a) como de los planteamientos que hicieron los directivos presentes en el "Foro de Consulta de Directivos de Escuelas Normales" celebrado el 17 de agosto de 2016. En general, se reconocen estas ideas en el segundo texto del modelo, si bien se considera necesario atender las inquietudes de los directivos que tienen que ver directamente con la mejora de los procesos formativos y con trazar un futuro estratégico para las instituciones formadoras de docentes.

La nueva administración federal ha establecido, a través del secretario de Educación Pública, Esteban Moctezuma Barragán, que el fortalecimiento de las escuelas normales del país es uno de los temas que se atenderá en el sexenio, ya que las considera "la base de todo el proceso educativo" (Excelsior, 2018, párr. 4), por lo tanto, se les destinará un mayor presupuesto. Además, en el dictamen de Reforma Educativa publicada en marzo de 2019, se establece que "El Estado fortalecerá a las instituciones públicas de formación docente, de manera especial a las escuelas normales, en los términos que disponga la ley" (Proyecto de decreto, 2019: 2).

Sin bien aún no se define cuáles serán las estrategias para el fortalecimiento de las escuelas normales en este sexenio, podría ser de interés para definirla considerar lo planteado por los directivos normalistas, que no fue recuperado en el Modelo Educativo 2017. Asignar a estas instituciones formadoras de docentes responsabilidades en materia de formación continua contribuiría a la transformación de las normales y, como fue mencionado en las mesas de trabajo, a su consolidación como IES. Además, la habilitación de su planta académica representa - de acuerdo a las demandas de los directivos - una línea de acción relevante para contribuir al fortalecimiento del servicio educativo que ofrecen. Resultaría pertinente que el planteamiento de la agenda educativa en este ámbito considerara las propuestas enunciadas por los actores que se encuentran más cercanos a la vida cotidiana de las escuelas normales, ya que son quienes conocen las necesidades y áreas de oportunidad que requieren atención.

\section{Referencias}

Abellán, J. (2016). "El modelo educativo 2016, entre la simulación y el falso consenso". Revista Latinoamericana de Estudios Educativos, 46(3), 45-84. México.

Cáceres, P. (2003). "Análisis cualitativo de contenido: una alternativa metodológica alcanzable". Psicoperspectivas, 2, 53-82. 
Diario Oficial de la Federación (2013a). Decreto por el que se reforman, adicionan y derogan diversas disposiciones de la Ley General de Educación. http://dof.gob.mx/nota detalle.php?codig $\mathrm{o}=5313841 \& \mathrm{fecha}=11 / 09 / 2013$

(2013b). Programa sectorial de educación 2013-2018. http://www.dof.gob.mx/nota detalle.php?codigo $=5326569 \&$ fecha $=13 / 12 / 2013$

(2013c) Ley General del Servicio Profesional Docente. www.dof.gob.mx/nota detalle.php?c odigo $=5313843 \&$ fech $a=11 / 09 / 2013$

Excelsior (diciembre, 2018). "Próximo gobierno va por rescate de escuelas normales: Esteban Moctezuma". Excelsior. https://www.excelsior.com.mx/nacional/proximo-gobierno-va-porrescate-de-escuelas-normales-esteban-moctezuma/1271306

Figueroa, L. (2000). “La formación de docentes en las escuelas normales: entre las exigencias de la modernidad y las influencias de la tradición". Revista Latinoamericana de Estudios Educativos, 30(1), 117-142. México.

Strauss, A. y J. Corbin (2002). Bases de investigación cualitativa. Técnicas y procedimientos para desarrollar la teoría fundamentada. Medellín: Universidad de Antioquía-Contus.

Instituto Electoral de Participación Ciudadana (s.f.). Mecanismos de participación ciudadana, Instituto Electoral de Participación Ciudadana. http://www.iepcjalisco.org.mx/mecanismos-participacion-ciudadana

Mayring, P. (2000). "Qualitative Content Analysis. Forum: Qualitative Social Research, 1(2).

Ornelas, C. (2013). "Si se da marcha atrás en reforma educativa, Peña Nieto se acaba". Siempre!. http://www.siempre.com.mx/2013/09/si-se-da-marcha-atras-en-reforma-educativa-penanieto-se-acaba/

Pacto por México (2012). http://www.foroconsultivo.org.mx/documentos/politicas publicas/ pacto por mexico.pdf

Pérez, M. (2015). "¿Quién es el docente universitario de la educación normal?". Revista Latinoamericana de Estudios Educativos, 45(3), 119-138. México. http://www.redalyc.org/articulo. oa?id=27041543005

Programa Interdisciplinario sobre Política y Prácticas Educativas - PIPE (2016). Consulta sobre el Modelo Educativo 2016. México: CIDE. https://framework-gb.cdn.gob.mx/happenings/ modelo-educativo-sep/05 Informe ME2016.pdf

Proyecto Estado de la Nación (2001). Auditoría ciudadana sobre la calidad de la democracia. Nueva York: PNUD.

Proyecto de decreto por el que se abrogan las diversas disposiciones de la "Reforma Educativa 2013" contenidas en la Constitución y sus respectivas leyes secundarias, que modifican los artículos 30, 31 y 73 de la Constitución Política de los Estados Unidos Mexicanos. (2019). https://interactivo.eluniversal.com.mx/online/pdf-19/REFORMAEDUCATIVA.pdf 
Reimers, F. (2011). "Participación ciudadana en reformas de políticas educativas". Pensamiento Educativo. Revista de Investigación Educacional Latinoamericana, 17(2), 115-131.

Scott, D., C. Posner, C. Martin y E. Guzman (2015). Interventions in Education Systems. Reform and Development. Nueva York: Peter Lang.

Secretaría de Educación Pública (2014). Comunicado 010. Anuncia el avance de Consulta Nacional para la Revisión del Modelo Educativo. https:/www.gob.mx/sep/prensa/comunicado-010-sep-anuncia-el-avance-de-consulta-nacional-para-la-revision-del-modeloeducativo?state=published

(2016a). Modelo educativo 2016. México: Autor. https://www.gob.mx/modeloeducativo2016

(2016b). Los fines de la educación del siglo XXI. México: Autor. (2017a). Modelo Educativo para la Educación Obligatoria. México: Autor. (2017b). Estrategia de Fortalecimiento y Transformación de las Escuelas Normales. Resumen Ejecutivo. México: Autor. https://docs.google.com/gview?url=https://www.gob.mx/cms/ uploads/attachment/file/240634/1.- Resumen Ejecutivo 7 .pdf

Zebadúa, E. (2013). "Reforma educativa en México". La crónica de hoy. http://www.cronica.com. $\mathrm{mx} /$ notas/2011/556315.html 